\title{
Microfluidic synthesis of metal oxide nanoparticles via the nonaqueous method
}

\author{
Pierre Stolzenburg $^{\mathrm{a} \dagger}$, Thomas Lorenz ${ }^{\mathrm{b} \dagger}$, Andreas Dietzel ${ }^{\mathrm{b}, \mathrm{c}}$, Georg Garnweitner ${ }^{\mathrm{a}, \mathrm{c}, *}$ \\ ${ }^{a}$ Institute for Particle Technology, Technische Universität Braunschweig, Volkmaroder Str. 5, D- \\ 38104 Braunschweig \\ ${ }^{b}$ Institute of Microtechnology, Technische Universität Braunschweig, Alte Salzdahlumer Str. 203, D- \\ 38124 Braunschweig \\ ${ }^{c}$ Laboratory for Emerging Nanometrology, Technische Universität Braunschweig, Langer Kamp 6B, \\ D-38106 Braunschweig \\ ${ }^{\dagger}$ The authors contributed equally to this study \\ *Corresponding author
}



\begin{abstract}
Microfluidic synthesis allows for a good control of the particle formation conditions while minimizing the consumption of material. In this study, we exploited these advantages for the nonaqueous synthesis of $\mathrm{TiO}_{2}, \mathrm{ZnO}$ and $\mathrm{CeO}_{2}$ nanoparticles in a closed micro droplet reactor which resulted in well-defined particle structures. Monodisperse droplets are generated in microfluidic flow-focusing area and
\end{abstract}


subsequently transported into a reaction zone allowing synthesis temperatures of up to $200{ }^{\circ} \mathrm{C}$. In addition, we showed that a continuous particle generation process using solid precursor species can be realized without channel clogging. We present different microfluidic designs with reaction zones consisting either of a long meandering channel or a short expansion channel to realize the different reaction times required for the respective model systems. Interestingly, we found that at elevated temperatures, the reaction mixture diffuses into the surrounding continuous phase fluid which leads to a shrinkage of the droplets, forcing eventually the assembly of highly spherical aggregate structures. Depending on the different model systems, we obtained nanospheres of about $100 \mathrm{~nm}$ from the $\mathrm{CeO}_{2}$ synthesis and microspheres up to $17 \mu \mathrm{m}$ in size from the $\mathrm{TiO}_{2}$ synthesis. In contrast, $\mathrm{ZnO}$ syntheses at milder temperatures were conducted without any droplet shrinkage which resulted in randomly shaped aggregates of $150 \mathrm{~nm}$ that self-assembled in the droplet micro reactor. Thus, a versatile synthesis technique is presented that can be applied for various metal oxides over a broad range of reaction parameters, as a promising approach for the controlled production of high quality nanoparticles.

\section{Keywords}

Microfluidic synthesis; droplet reactors; multiphase processing; flow focusing; nonaqueous synthesis; metal oxide nanoparticles

\section{Introduction}

Nanostructured metal oxides (Niederberger et al., 2005; Pinna et al., 2011) such as titanium dioxide (Barth et al., 2015; Bayani et al., 2016), zinc oxide (Luo et al., 2013; Segets et al., 2009) and cerium dioxide (Chen and Xu, 2018; Seong et al., 2018; Song et al., 2018) are promising materials for current and future applications as catalysts (Low et al., 2018; Wang et al., 2017b), sensors (Ha et al., 2018; Uzunoglu and Stanciu, 2016), nanocomposites (Khataee et al., 2018; Mallakpour and Shamsaddinimotlagh, 2018) or functional ceramics (Bills et al., 2015; Lu et al., 2017). The nonaqueous sol-gel method is a versatile synthesis route to fabricate these materials in a highly 
crystalline form with well-defined properties (Bilecka et al., 2011; Garnweitner et al., 2008; Niederberger et al., 2002). Various works have unraveled the fundamental phenomena behind particle formation during the nonaqueous synthesis (Jensen et al., 2010; Ludi et al., 2012; Niederberger et al., 2005), whereby it has been shown that a thorough knowledge of the molecular mechanisms is necessary to understand the complex interplay between synthesis conditions and final product properties (Deshpande et al., 2005; Koczkur et al., 2015; Olliges-Stadler et al., 2013). Based on this expertise, it is feasible to design processes that are able to customize size, morphology as well as phase composition of the resulting nanoparticles. We have shown that the nonaqueous synthesis can be adapted to tailor the nanoparticle properties of titanium dioxide (Zimmermann et al., 2013), aluminum zinc oxide (Zellmer et al., 2015) and zirconium dioxide (Cheema et al., 2012) on a larger process scale using batch reactor systems. Yet, a continuous processing technique would be desirable for enabling the large-scale synthesis with highest efficiency and stability to supply the rising demand of customized metal oxide nanoparticles with high production rates at low cost (Tsuzuki, 2009). Whilst continuous gas phase processes using e.g. flame pyrolysis (Gröhn et al., 2014; Jossen et al., 2006; Mueller et al., 2003) and hot wall reactors (Ruusunen et al., 2015) are widely applied, a continuous wet chemistry approaches are much less performed (Gruar et al., 2013; Nishioka et al., 2011; Slostowski et al., 2012), although they stand out through milder synthesis conditions and a relatively facile control of particle sizes. A major challenge for the production of metal oxide nanocrystals at larger scale is the realization of homogenous synthesis conditions in the reaction vessel, which is indispensable to achieve a uniform product. Microfluidic processing facilitates the fulfillment of these demands as small reaction volumes allow fast heat transfer at laminar flow and thereby good controllability of the synthesis parameters (Ralf et al., 2012; Song et al., 2006). Moreover, microfluidic systems can be operated continuously and are feasible to yield larger massfluxes when parallelized. In the last years, microfluidics became a highly interesting platform for the preparation and production of micro- and nanoparticles (Chang et al., 2008; Shi et al., 2017; Erfle et al., 2017). Whilst typically, room temperature or slightly elevated temperatures are utilized for experimental simplicity, a few works have investigated nanoparticle synthesis at high temperatures in 
organic media (Marre et al., 2008; Nightingale et al., 2011). As an example, a segmented flow approach suitable for high temperatures was introduced by Yen et al. (2005) who showed that gasliquid separated flow overcomes slow diffusive mixing conditions which leads to uniform residence time and narrow size distributions. Hence, by performing the synthesis in a micro droplet reactor, the chemical reaction is handled in a secluded volume in a highly controlled manner with minimal material loss (Lee et al., 2012). Furthermore, droplet microfluidic processing enables the formation of extremely well-defined emulsions, since precise application of energy is achieved during droplet formation. Thus, the prepared droplets can be used as templates and reaction containers for micro- and nanoparticles during the particle formation reaction (Wang et al., 2017a; Zhang et al., 2016). Khan et al. conducted a silica droplet synthesis in a microfluidic system and obtained spherical colloids with sizes in the submicron regime (Khan et al., 2004). Further studies exploited that the liquid-liquid interface of droplets can act as a template to synthesize iron oxide nanoparticles, whereas a controlled hierarchical assembly of primary particles can be achieved through macromolecules (Sophia Lee et al., 2012). In our previous work, a 3D flow focusing device made from glass was used to precipitate highly monodisperse nanoparticles in droplets (Lorenz et al., 2018). The nonaqueous sol-gel method to the best of our knowledge has only in one report been described to be carried out in a microfluidic system. Koziej et al. (Koziej et al., 2013) synthesized tungsten oxide nanoparticles while heating the reaction mixture with a microwave device and investigated the temperature evolution in the droplets with in situ IR-spectroscopy. Koziej et al. showed that fast-heated droplets bearing a homogeneous temperature result in homogenous formation and growth conditions, achieving rather uniform and crystalline particles. In this study, we attempt to establish the nonaqueous synthesis in microfluidic systems with large applicability for a broad range of nanostructured metal oxides with a focus on process engineering aspects. Thus, we concentrate on a precise control of the synthesis conditions throughout the entire reaction system in combination with a steady and stable processing over the course of longer experiments to yield larger product quantities. In addition, we present different microfluidic setups that are suitable for synthesis demanding longer and shorter reaction times at higher reaction temperatures. 


\section{Materials and methods}

\subsection{Microfluidic device \& experimental setup}

The used microfluidic emulsification devices enable the droplet generation of the reaction mixture in a continuous phase fluid. The devices are designed with a flow-focusing geometry as described by Anna et al. (Anna et al., 2003). Two continuous phase channels encounter the channel of the disperse phase orthogonally from both sides and focus the disperse phase fluid into a constriction. The disperse phase channel and the emulsion channel behind the constriction have a width of $100 \mu \mathrm{m}$ at the intersection (see Fig. 1.a). Continuous phase channels and the constriction possess a width of $50 \mu \mathrm{m}$. The constriction length is $50 \mu \mathrm{m}$, whereby all structures exhibit a height of $100 \mu \mathrm{m}$. The heatable reaction zones are located at a distance of $3 \mathrm{~mm}$ behind the flow-focusing geometry. Two different microfluidic designs were used during the experiments: The first design features a meandering channel at the heated reaction zone as shown in Fig. 1.b. The channel has a width of $300 \mu \mathrm{m}$ and a total length of $450 \mathrm{~mm}$ inside the heated region. This geometry was designed to separate all droplets from each other after droplet formation and avoid coalescence. The second design is shown in Fig. 1.c and features a reaction zone with a channel of $3 \mathrm{~mm}$ in width, in order to decrease the flow velocity and achieve a long residence time of the droplets in the heated area. This geometry was introduced after establishing a good stability of the droplets against coalescence by adding a fluorinated surfactant (see section 2.2). The devices are made of PDMS by soft lithographic fabrication methods as described by McDonald and Whitesides (McDonald and Whitesides, 2002). Negative masters were fabricated using the SU-8 2000 series photo resists. One $100 \mu \mathrm{m}$ thick layer of SU-8 2050 was spin-coated onto the glass substrate followed by a soft bake step. Exposure was performed using a mask aligner (EVG 620, ECG Group) and a photolithographic mask. A post-exposure bake was carried out prior to development of the structures. PDMS (SYLGARD 184, Dow Corning) molding was subsequently done using the obtained SU-8 masters. Cured systems were capped using unstructured PDMS slabs to 
ensure the same material and wetting properties at all channel walls. The systems were placed on glass slides for better handling and precise placement in the temperature regulated mounting.
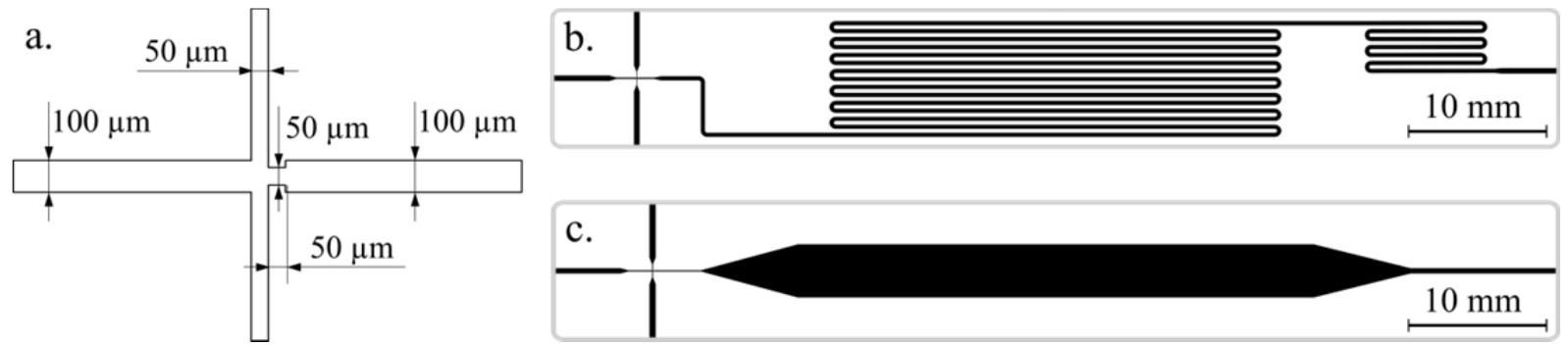

Fig. 1: Schematic of the microfluidic flow-focusing devices: a. shows the flow-focusing geometry used in all devices; b. Microfluidic design with meandering channel in the heated reaction zone; c. Microfluidic design with expansion channel in the heated reaction zone.

Another core element of the experimental setup is the aluminum mounting for the microfluidic chip (see Fig. 2). The mounting features a heating element $(800 \mathrm{~W})$ that consists of an aluminum block with four heat cartridges and is located below the reaction zone of the microfluidic chip. The heat cartridges are controlled using a feedback loop temperature regulator with a thermocouple. Actively cooled elements are located at both sides of the heating element. Water circulation is used to keep the elements at a constant temperature. Cooled and heated aluminum elements are thermally insulated from each other by ceramic distance bushes. The mounting is attached onto a movable stage and placed under a microscope (Stemi 508, Zeiss) with a camera (acA2040-90uc, Basler) for optically monitoring the emulsification and precipitation processes. 


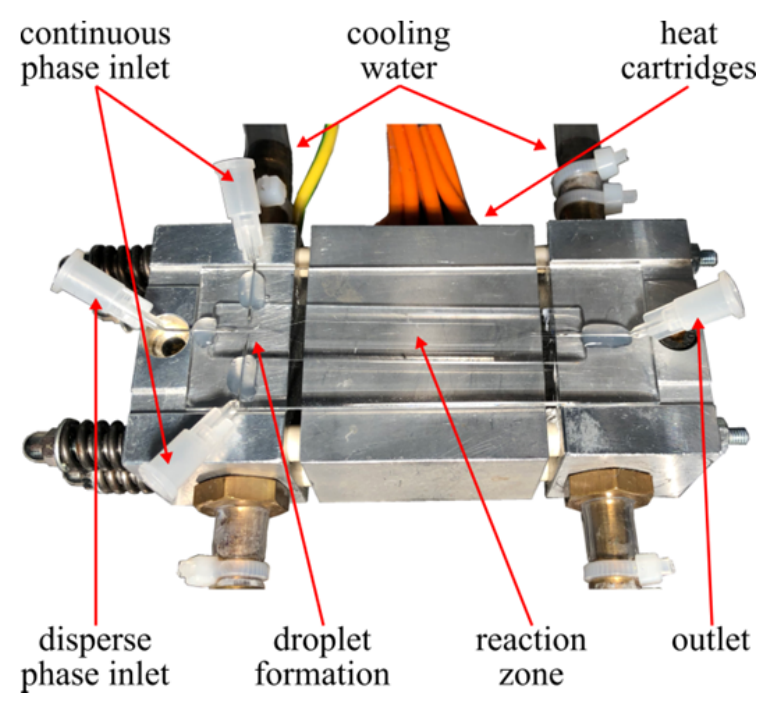

Fig. 2: Photograph of the temperature controlled mounting with microfluidic chip.

The complete measurement setup is shown in Fig. 3.Electro-pneumatic regulators (EPR) (ITV0051, SMC) were connected to the supply vessels containing the continuous phase fluid (CP) and the disperse phase fluid (DP), which were guided to the microfluidic chip using PTFE tubing. The tubing of the continuous phase was split in two equally long parts for the connection of both continuous phase channels. The outlet of the microfluidic chip was connected to a collecting vessel. For experiments with $\mathrm{ZnO}$ and $\mathrm{CeO}_{2}$ the collecting vessel was placed on a hot plate and actively stirred by a magnetic stirrer and is termed semibatch reactor (SBR) in the following.

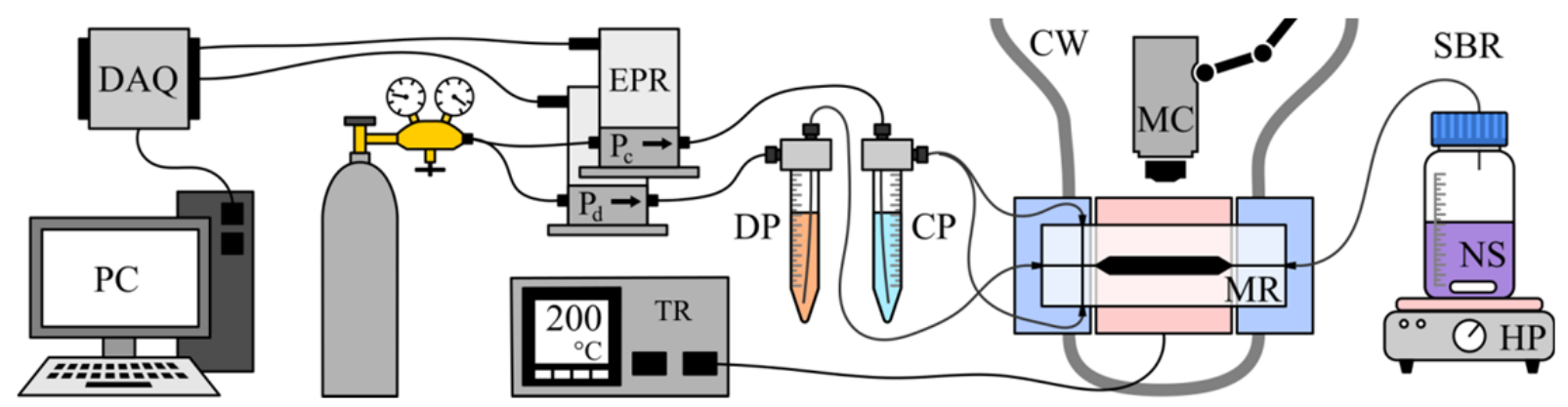

Fig. 3: Schematic of the experimental setup. Blue colored areas of the device mounting are cooled by circulating water. The red colored area is heated using heat cartridges and a temperature regulator. DAQ $=$ data acquisition module, $\mathrm{EPR}=$ electro-pneumatic regulator, $\mathrm{TR}=$ temperature regulator, $\mathrm{DP}=$ disperse phase fluid, $\mathrm{CP}=$ continuous phase fluid, $\mathrm{CW}=$ cooling water, $\mathrm{MC}=$ microscope with camera, $\mathrm{MR}=$ microfluidic reactor, 
$\mathrm{SBR}=$ semibatch reactor, $\mathrm{NS}=$ nanoparticle suspension, $\mathrm{HP}=$ hot plate with magnetic stirrer (not used for experiments with $\mathrm{TiO}_{2}$ ).

\subsection{Materials and experimental conditions}

A typical synthesis of the model systems $\mathrm{ZnO}, \mathrm{TiO}_{2}$ and $\mathrm{CeO}_{2}$ comprised preparing the disperse phase (DP) by dissolving and mixing the precursor species zinc acetylacetonate hydrate (powder, $\mathrm{Zn}(\mathrm{acac})_{2}$, Sigma-Aldrich), titanium(IV) isopropoxide (liquid, $\mathrm{Ti}(\mathrm{OiPr})_{4}, 97 \%$, Sigma-Aldrich) or cerium trichloride (powder, Sigma-Aldrich, $\left.\mathrm{CeCl}_{3}\right)$ with the solvent benzyl alcohol $(\mathrm{BnOH}, 97 \mathrm{wt} \%$, Merck) in a flask. A standard precursor concentration of $180 \mathrm{mmol} \mathrm{L}^{-1}$ was used throughout all syntheses. The mixtures containing $\mathrm{Zn}(\mathrm{acac})_{2}$ and $\mathrm{CeCl}_{3}$ were heated to $70{ }^{\circ} \mathrm{C}$ under agitation to dissolve the precursor species. Even though the precursor species seemed to be completely dissolved, the resulting precursor solutions were filtered to provide a homogenous reaction mixture in order to prevent clogging of the channels. Afterwards, the solution was stored in the heated DP vessel and supplied to the device through heated tubing while operating the emulsification zone at $70{ }^{\circ} \mathrm{C}$ (Fig. 3) to prevent recrystallization of the precursor species prior to reaching the reaction zone. The synthesis was then conducted in a single microfluidic device for up to 6 hours without clogging, including refills of the supply vessels with the CP and DP.

The nanoparticle syntheses were performed in microfluidic droplets containing the reaction mixture. These droplets were generated in the microfluidic emulsification device using the fluorocarbon oil Fluoronox 1008 (TECCEM GmbH) as the continuous phase fluid, focusing the reaction mixture into the orifice. A specially synthesized fluorinated surfactant reagent was previously added to the CP to initiate droplet formation as well as stabilizing the droplets against coalescence. The surfactant was synthesized using the route of Holtze (Holtze et al., 2008). A perfluoro-polyether "Krytox 157-FSH" (Dupont) with a carboxylic group at the end was activated with oxalyl chloride (Sigma-Aldrich) (Lee et al., 2014) before coupling a 1,6-hexanediamine (Sigma-Aldrich) to the fluorinated block which resulted in a surface active diblock molecule. Droplet formation appeared in the dripping regime resulting in monodisperse droplets. The diameter of the detaching droplets was 
between 50 and $100 \mu \mathrm{m}$ depending on the model system and the applied pressures. To maintain proper droplet formation during the transition from initial priming to the heat-up phase, the applied pressures had to be adjusted, due to the temperature dependency of the viscosity of the fluid and the resulting change in hydraulic resistance and flow rate. This effect was more pronounced in the meandering systems, as the overall channel cross-section is much smaller than for the expansion systems. Once the final temperature had been reached, steady state of the flow rates appeared and no further adjustment of operation pressures was needed.

After emulsification, the droplets flowed downstream into the reaction zone which was operated at temperatures ranging from 100 to $200{ }^{\circ} \mathrm{C}$. The residence time in the reaction zone ranged from approximately $25 \mathrm{~min}$ for the meandering systems to $10 \mathrm{~min}$ for the expansion systems. Depending on the model system used, the reaction times of the different syntheses varied from minutes to hours, and the droplet residence time had to be adjusted for each model system to initiate particle formation within the droplets. Hence, we operated either a setup with long meandering channels (Fig. 1.b) or a setup with an expansion channel with a downstream stirred semi-batch reactor (SBR) (Fig. 3) for syntheses that required longer droplet residence times. For syntheses that can be performed with shorter droplet residence times, a system with solely the expansion channel was sufficient (Fig. 1.c). Nevertheless, before employing the synthesis in the microfluidic system, we evaluated the reaction times of the model systems by performing in situ turbidity measurements in a $250 \mathrm{~mL}$ steel reactor equipped with a laser-detector system which was designed for previous studies to investigate the particle formation mechanisms during the nonaqueous synthesis (Stolzenburg and Garnweitner, 2017). A representative reaction mixture was heated to process temperature and the turbidity was measured to detect the decrease in the transmission intensity during the synthesis (supplementary information Fig. S.2). We defined the time frame from reaching process temperature until the occurrence of the intensity decrease as the (minimum) reaction time for particle formation. Hence, we screened various precursor concentration - temperature combinations to find the process parameters that have reaction times shorter than the microfluidic system droplet residence times. 
The resulting emulsions $(\mathrm{CP}+\mathrm{DP})$ were centrifuged at $5000 \mathrm{~min}^{-1}$ to evoke a phase separation. As both phases contained nanoparticles, we analyzed the upper and lower phase individually. Furthermore, we dried the nanoparticle suspensions under vacuum to yield the solid phase.

\subsection{Characterization}

We investigated the solubility of the reaction mixture and the solvent in the continuous phase fluid at elevated temperatures $\left(80\right.$ to $200{ }^{\circ} \mathrm{C}$ ) by heating a mixture of both fluid phases under constant agitation. After two hours at a given temperature, we took a sample of the CP and cooled it down to ambient temperature which resulted in a phase separation of two fluid phases. As the CP had the higher density $\left(1.85 \mathrm{~g} \mathrm{~mL}^{-1}\right)$ compared to the DP $\left(0.96 \mathrm{~g} \mathrm{~mL}^{-1}\right)$, we measured the volume of the upper phase to determine the solubility. Furthermore, both phases showed no miscibility at ambient conditions, which therefore was neglected.

The droplet sizes as well as the droplet velocities were measured optically using pictures recorded by the microscope camera (see Fig. 3) and the image analysis software ImageJ (https://imagej.nih.gov/ij/). Furthermore, the average droplet residence time in the reaction zone was determined.

We analyzed the resulting nanoparticle suspensions in both phases (CP and DP) by dynamic light scattering (DLS) using a Zetasizer Nano ZS (Malvern Instruments). The particle sizes and structures were investigated by scanning electron microscopy (SEM, DSM 960 A, Zeiss) and transmission electron microscopy (TEM, JEM-2100F-UHR, JEOL). We performed selective area diffraction (SAED, JEM-2100F-UHR, JEOL) to investigate the crystallinity of the primary particles. Furthermore, the solid phase was investigated by powder X-ray diffraction $(\mathrm{Cu} \mathrm{K \alpha}$ radiation; Empyrean Cu LEF HR goniometer; Empyrean series 2, PANalytical, PIXcel-3D detector; Si wafer; $20-90^{\circ}$, step size $0.05^{\circ}$ ) and subsequent Rietveld refinement to determine the crystallite sizes and phase composition of the synthesized nanoparticles.

\section{Results and discussion}




\subsection{Microfluidic emulsification}

Various microfluidic emulsification devices, continuous phase fluids and surfactants were tested for the proper emulsification of the reaction mixtures for each model system. The main challenges can be explained by wettability problems. Fluorocarbon oils were desired as continuous phase fluids to avoid chemical reactions and miscibility between the disperse and continuous fluids. Thus, the resulting emulsion will be categorized as a water-in-oil emulsion rather than an oil-in-water emulsion. The material of the microfluidic channels should therefore exhibit a hydrophobic and lipophilic surface. PDMS is known to possess the desired wettability properties and therefore was chosen as construction material. Simple T-junction geometries were tested first for emulsification, but led to the adhesion of the disperse phase fluid to the channel walls and did not allow proper emulsification during longer experiments. Therefore, 3D flow-focusing as described by Anna et al. (Anna et al., 2003) was used to establish a stable emulsification of the DP in homogenous droplet sizes over the course of the entire synthesis. Furthermore, the droplet size was easily controllable by adjusting the operating pressures.

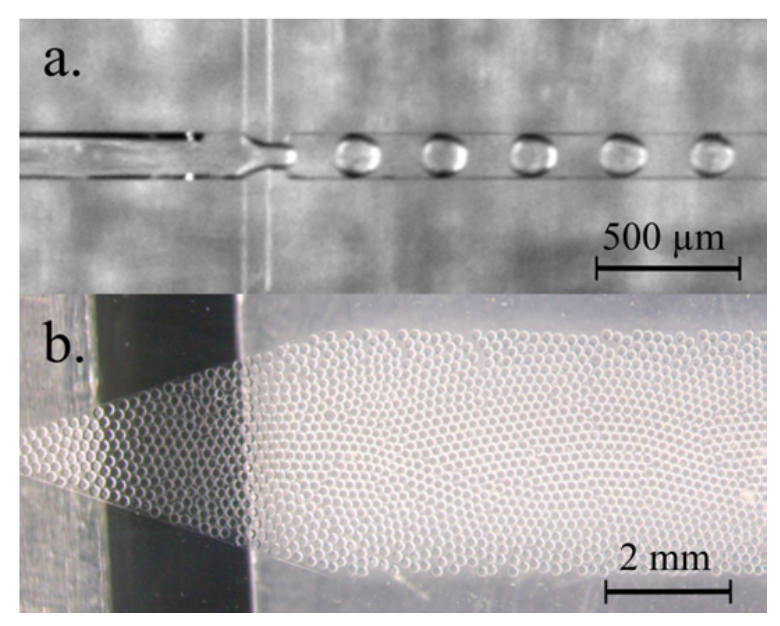

Fig. 4: Droplets inside the microfluidic device: a. Droplet formation of precursor and benzyl alcohol in Fluoronox; b. Stable and monodisperse droplets in the reaction zone of the device.

The emulsification zone and the reaction zone were separated to operate both zones at different temperatures, which was necessary as we observed that droplet rupture was not feasible at elevated temperatures above $100^{\circ} \mathrm{C}$. However, once formed, the droplets were stable against coalescence at high temperatures in the reaction zone. Interestingly, we observed shrinkage of the droplets while 
flowing downstream at higher temperatures as shown in Fig. 5.This can be explained by the miscibility of the reaction mixture in the fluorinated oil which we investigated through solubility measurements as shown in Fig. 6. We found that the solubility of benzyl alcohol in the fluorocarbon oil Fluoronox 1008 significantly increases at temperatures exceeding $120^{\circ} \mathrm{C}$, whereas the solubility of the reaction mixture containing the liquid precursor species $\mathrm{Ti}(\mathrm{OiPr})_{4}$ and isopropanol was already increased at $100{ }^{\circ} \mathrm{C}$. In fact, the higher solubility of the reaction mixture is due to the good solubility of isopropanol, which stems from a ligand exchange reaction that occurs in the solution at ambient temperature (Zimmermann and Garnweitner, 2012). A limited miscibility of fluorocarbons and aromatic compounds is well known as a thermodynamic consequence of low solubility parameters promoting a slight miscibility increase at higher temperatures (Scott, 1948). After all, this has a major impact on the properties of the particles synthesized at higher temperatures, i.e. for the $\mathrm{TiO}_{2}$ and $\mathrm{CeO}_{2}$ model systems, which we will discuss in more detail in section 3.2. As a consequence, the shrinkage of the droplets decreases the reaction volume and continues until the entire amount of the solvent is dissolved in the continuous phase fluid. Thus, the formed particles assemble into a spherical shape and are dispersed in a homogenous fluid phase as schematically shown in Fig. 5.a. After cooling, the temporally homogenous phase separates into the CP and DP, yielding an emulsion with dispersed particles. 


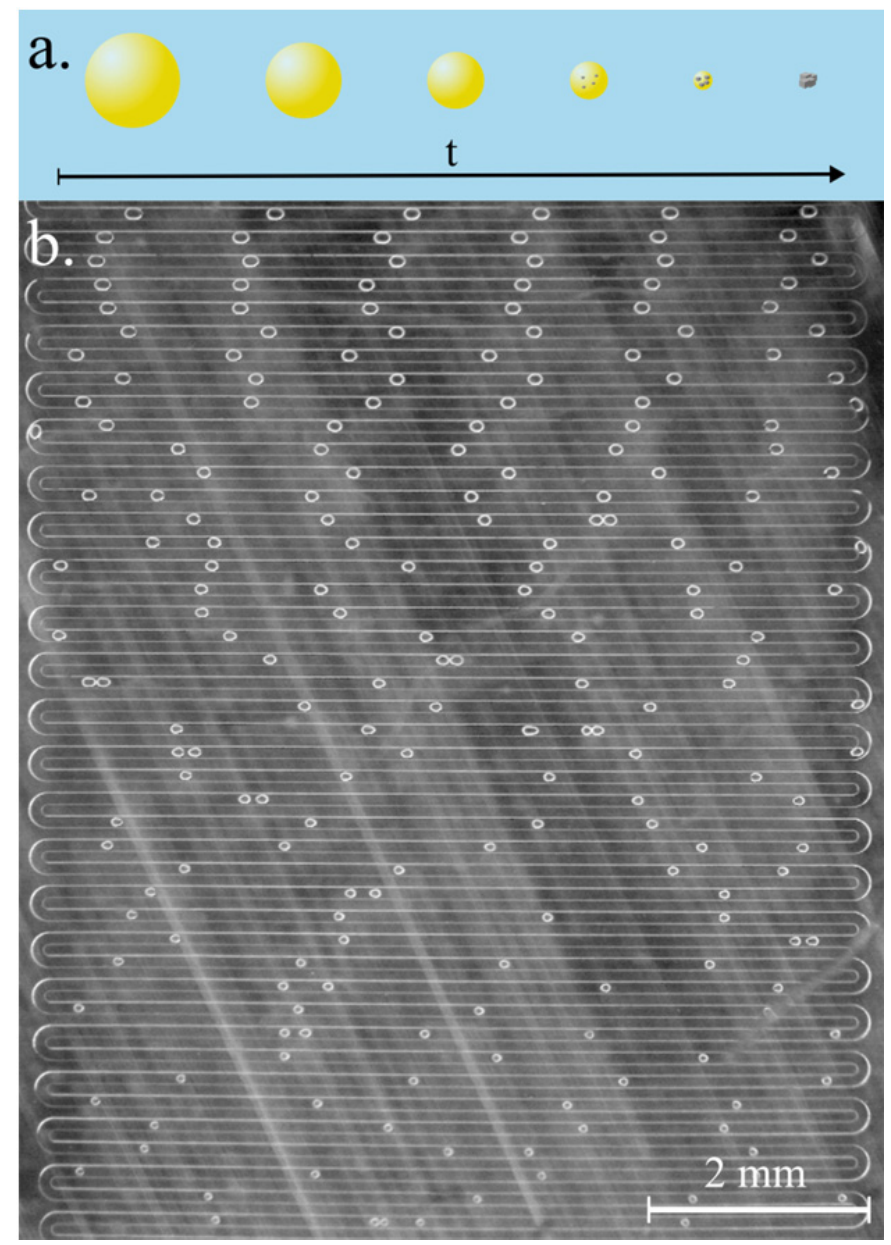

Fig. 5: Dissolution of benzyl alcohol in the continuous phase: a. scheme of droplet shrinkage over time; b. microscopic view showing shrinkage of droplets in meandering channels during a $\mathrm{TiO}_{2}$ synthesis performed at $200{ }^{\circ} \mathrm{C}$. 


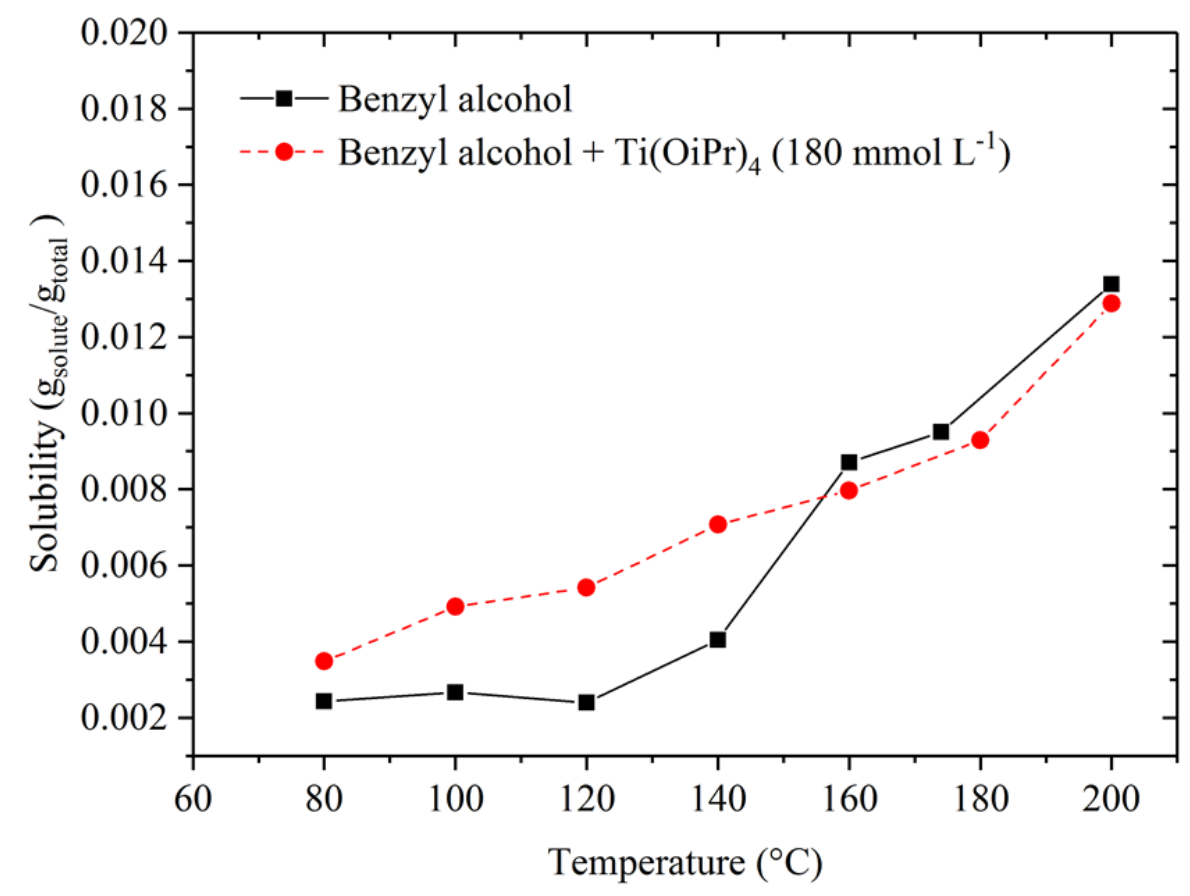

Fig. 6: Solubility of benzyl alcohol and the reaction mixture (titanium(IV) isopropoxide and benzyl alcohol) in the fluorocarbon oil Fluoronox 1008 showing that the miscibility increases at higher temperatures.

A full survey on the influence of flow rates has not been conducted in this study as we have found that the droplet residence time parameter can be correlated more easily to the actual reaction time within the micron-sized droplet reactors. Moreover, fluid motion was pressure driven using electropneumatic regulators and no further flow rate measurements have been performed in this setup. Nevertheless, we compared this experimental setup with a similar experimental setup using syringe pumps and found that equal droplet sizes and droplet residence times were obtained with flow rates ranging at $1-5 \mu \mathrm{L} \mathrm{min}{ }^{-1}$ for the disperse and $10-20 \mu \mathrm{L} \mathrm{min}^{-1}$ for the continuous phase fluid. Assuming similar volumetric flow rates for both phases in the pressure driven setup and a full conversion of the precursor species, one can estimate a quantitative yield of $5 \times 10^{-2} \mathrm{mmol} \mathrm{h}^{-1}$ for each model system.

\subsection{Synthesis of $\mathrm{TiO}_{2}$ nanoparticles}

We performed the $\mathrm{TiO}_{2}$ synthesis in the microfluidic device with the meandering channel at $200{ }^{\circ} \mathrm{C}$ with a precursor concertation of $180 \mathrm{mmol} \mathrm{L}^{-1}$. The emulsification zone was operated at lower temperatures corresponding to the temperature of the cooling water (around $15^{\circ} \mathrm{C}$ ) in order to realize a 
homogenous droplet formation during longer experiments. After emulsification, the droplets flowed downstream into the reaction zone which heated the droplets very fast to the reaction temperature. Upon reaching a temperature of $150{ }^{\circ} \mathrm{C}$, the benzyl alcohol solvent of the DP dissolved in the $\mathrm{CP}$ which caused the droplets to shrink while flowing through the meandering channel. This resulted in a concentration increase of the precursor species in the droplets which accelerated the particle formation reaction. In addition, fast reaction rates in a microfluidic synthesis are induced according to Cottam et al. by high local heat transfer rates and short diffusion lengths (Cottam et al., 2007). Furthermore, the shrinkage of the droplets leads to the formation of spherical aggregates as illustrated in the electron micrographs in Fig 7. The resulting microspheres had an average size of $13 \mu \mathrm{m}$ (see also Supplementary Information section, Fig. S.3), whereby the relatively homogenous size can be related to the steady process conditions realized through the microfluidic synthesis concept. Besides, the surfactant fosters the formation of the spherical structures by preventing a coalescence of the shrinking droplets as wells as enabling the stabilization of the aggregates against further agglomeration. Zhao et al. conducted an aqueous $\mathrm{TiO}_{2}$ nanoparticle synthesis within a droplet micro reactor without shrinkage and observed that spherical structures form when high precursor concentrations are used (Zhao and Middelberg, 2014). Well-defined microspheres were also fabricated from a droplet-based microfluidic silica synthesis by Carroll et al. (Carroll et al., 2008), who showed in a similar fashion to our synthesis that the globular structure arises from the solvent being removed from the droplet. Moreover, the surfactant molecules played a key role as they stabilized the droplet interface as the particles condensed inside the droplet, which eventually led to the final spherical shape.

Scanning- and transmission electron microscopy (Fig 7) shows that the $\mathrm{TiO}_{2}$ aggregates possess a coarse surface which results from randomly and densely packed primary nanoparticles with a size of about 6-7 nm (Fig 7.a) that form pores on the surface. The formation of pores during the synthesis of titania microspheres has been studied by Fischer et al. (Fischer et al., 2017), who state that pore formation is due to ligand species that are bound to the particle surface and prevent local crystallization. Hence, we suggest that in a similar fashion, the involved surfactant species might bind to the particle surface and prevent the formation of compact aggregates of primary particles, hindering 
the formation of a smooth surface. Thus, we suggest that the nucleated nanoparticles instantly aggregate and are forced by the shrinking droplet to form spherical structures with a coarse surface.

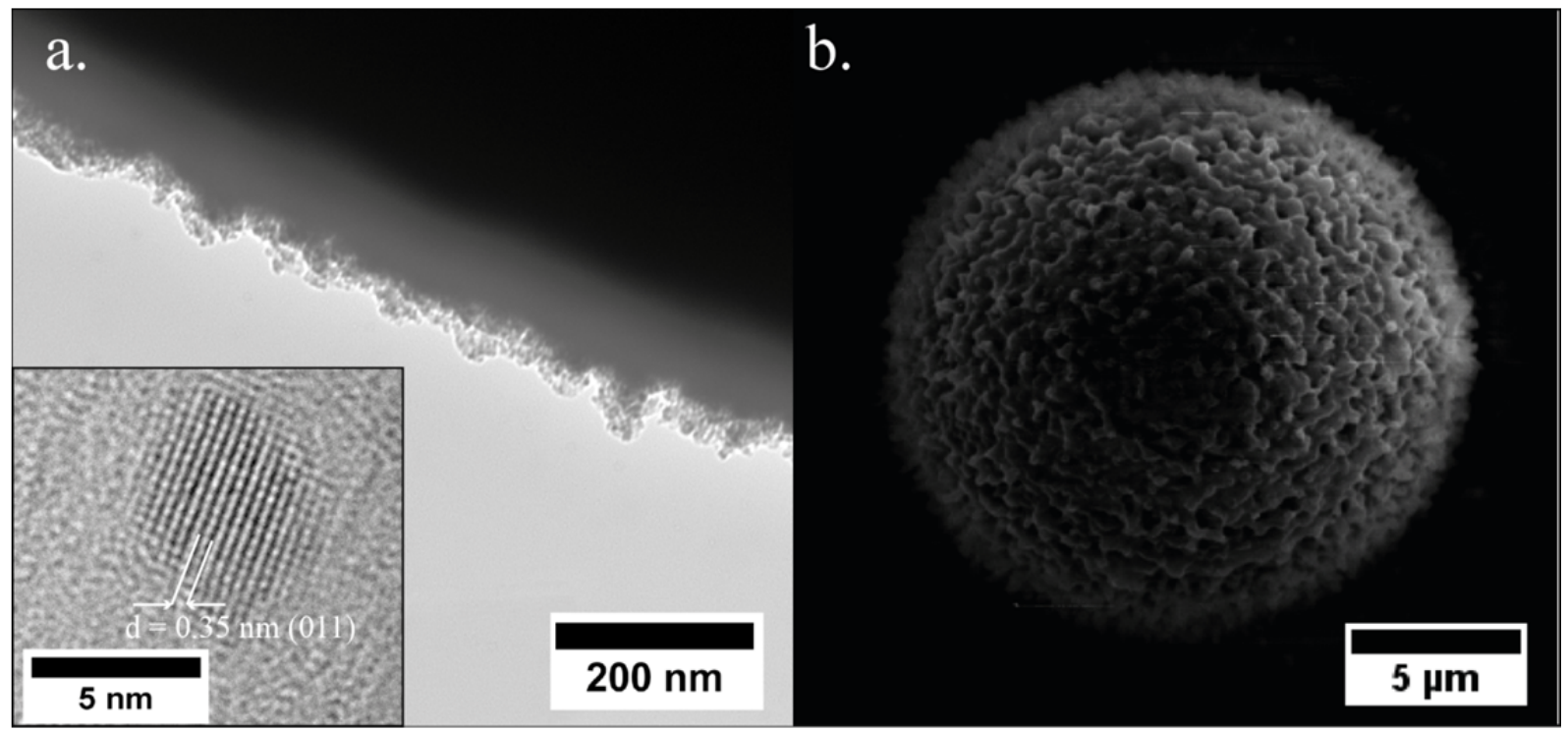

Fig 7: a. Transmission electron micrograph of the interface of a spherical $\mathrm{TiO}_{2}$ aggregate as obtained from a synthesis conducted in a microfluidic device using the meandering channel at $200{ }^{\circ} \mathrm{C}$ with a precursor concentration of $180 \mathrm{mmol} \mathrm{L}^{-1}$. Inset: magnification showing a $\mathrm{TiO}_{2}$ primary nanoparticle of about $6.5 \mathrm{~nm}$ in size with a lattice spacing $d$ of $0.35 \mathrm{~nm}$ corresponding to the (011) lattice plane of the anatase polymorph. $\mathrm{b}$. Scanning electron micrograph of a spherical $\mathrm{TiO}_{2}$ aggregate obtained at the same conditions.

The suspension was dried under vacuum at $40{ }^{\circ} \mathrm{C}$ for 24 hours which yielded a highly viscous paste-like organic matrix with embedded $\mathrm{TiO}_{2}$ particles. We were not able to recover sufficient quantities after particle washing and thus we investigated the nanoparticles within the organic matrix by XRD measurements, whereby the high organic content caused the relatively high noise level in the measurement. Fig. 8 shows the diffractogram of a sample synthesized at $200{ }^{\circ} \mathrm{C}$, whereby we determined a crystallite size of $6.3 \mathrm{~nm}$ from the reflection of the (011) lattice plane which is in great accordance with the crystallite size obtained from TEM analysis (Fig 7.a). In addition, the XRD analysis revealed that the particles consist of the anatase polymorph which typically forms in the nonaqueous synthesis in benzyl alcohol (Garnweitner and Niederberger, 2008). In contrast to our 
study, Zimmermann et al. (Zimmermann and Garnweitner, 2012) conducted the synthesis in a closed batch reactor system and observed that the nanoparticles nucleate at a size of $6 \mathrm{~nm}$ and then grow to $14 \mathrm{~nm}$. This indicates that nanoparticle growth seems to be suppressed when performed in a micro reactor. However, the reaction times in the microfluidic system were much shorter compared to the batch reactor system, which might result in a termination of the reaction before extensive nanoparticle growth can occur.

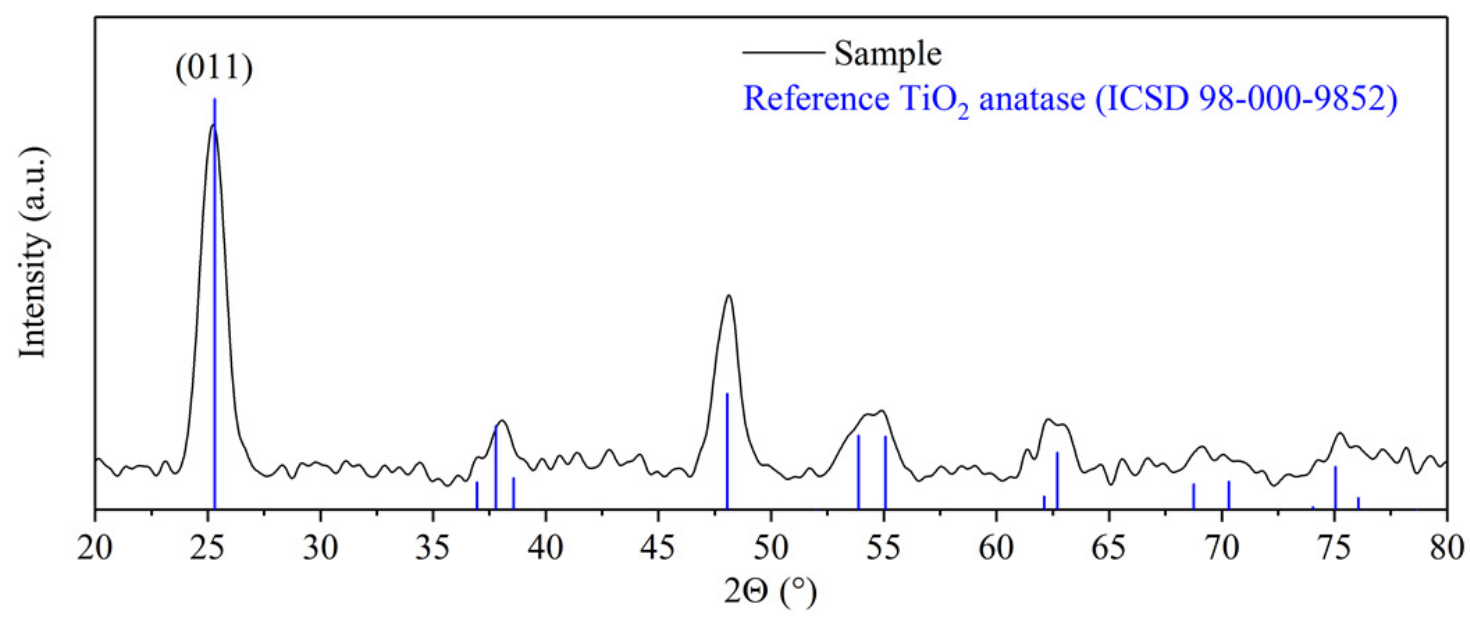

Fig. 8: Powder XRD pattern of a sample synthesized in droplets at $200{ }^{\circ} \mathrm{C}$, showing that the resulting particles are crystalline and consist of the anatase $\mathrm{TiO}_{2}$ polymorph (reference ICSD 98-000-9852)

We furthermore conducted the synthesis in the microfluidic system with the expansion channel and used a downstream semibatch reactor (SBR) to extend the residence time of the droplets in the reaction zone. Thereby, the heating zone was connected via tubes to a temperature resistant $20 \mathrm{ml}$ laboratory wide-mouth glass bottle which was constantly stirred and heated through a heating plate (Fig. 1). Although the resulting emulsion was stirred for up to 6 hours, we obtained the same primary particles sizes of $6 \mathrm{~nm}$, which was contrary to our expectation that further particle growth would occur due to the longer reaction times. In contrast to Zimmermann et al., the microfluidic synthesis was conducted using a downstream SBR as an open reaction system and consequently operated under ambient pressure. As higher pressures, caused by the vapor-liquid-equilibrium at elevated temperatures in a closed reaction system, have shown to influence the reaction mechanisms as well as 
reaction kinetics (Zimmermann et al., 2013), the low pressures and different synthesis conditions in the SBR might prevent particle growth. The downstream SBR results in similar spherical particle structures as described above, showing that the expansion channel - SBR system yields the same nanoparticle product as the meandering channel system. From a process perspective, the expansion channel - SBR system is preferred as it is less prone to particle clogging and thus more suitable for long time operations. Moreover, the SBR can be easily replaced by a continuous stirred tank reactor (CSTR) and thereby operated as within a continuous process, which makes a throughput scale-up through parallelization feasible.

\subsection{Synthesis of $\mathrm{ZnO}$ nanoparticles}

Compared to the $\mathrm{TiO}_{2}$ model system, the formation of $\mathrm{ZnO}$ nanoparticles during the nonaqueous synthesis occurs faster and demands lower temperatures (Zellmer et al., 2015), which enabled us to conduct the synthesis within the microfluidic setup using an expansion channel for short droplet residence times (Fig. 11.c). As we have observed particle formation in the droplets (Fig. 9) half-way through the expansion channel when the synthesis was conducted at $100{ }^{\circ} \mathrm{C}$, we investigated the synthesis in a temperature range from 100 to $150{ }^{\circ} \mathrm{C}$ with a precursor concentration of $180 \mathrm{mmol} \mathrm{L}{ }^{-1}$. The precursor solution was kept at $70{ }^{\circ} \mathrm{C}$ through external heating of the DP vessel and the tubes connecting the emulsification zone. Recrystallization of the precursor species in the emulsification zone was suppressed by operating the zone at temperatures between 70 to $80^{\circ} \mathrm{C}$ through regulating the cooling water supply. This enabled us to operate the microfluidic device under stable conditions up to 6 hours without clogging of the tubes, which was necessary to obtain a sufficient quantity for the subsequent analysis and characterization of the synthesized nanoparticles. Afterwards, the reaction mixture droplets flowed through the reaction zone without shrinkage as the solubility of the reaction mixture in the continuous phase fluid for these temperatures was relatively low (Fig. 6). 


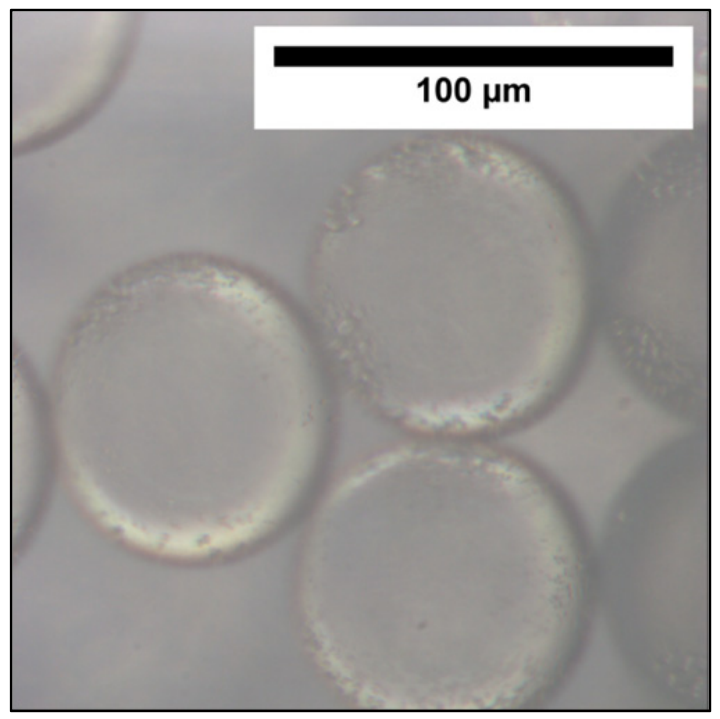

Fig. 9: Droplets of the reaction mixture containing $\mathrm{ZnO}$ particles synthesized at $130{ }^{\circ} \mathrm{C}$.

The droplets flowed into the storage vessel after passing the reaction zone which resulted in an emulsion of the continuous and the disperse phases. The fluid phases were separated by centrifugation, whereby both phases contained nanoparticles which were stable in solution. We investigated the nanoparticle suspensions via dynamic light scattering and found no significant differences in particle size between the respective suspensions. Fig. 10.a illustrates the size distributions of particles in the $\mathrm{CP}$ that were synthesized at 100,130 and $150{ }^{\circ} \mathrm{C}$. Interestingly, all size distributions showed a particle size $\mathrm{x}_{50,3}$ of $145 \pm 7 \mathrm{~nm}$ which suggests only a minor influence of the synthesis temperature. Transmission electron microscopy from a sample synthesized at $130{ }^{\circ} \mathrm{C}$ showed particles aggregates with random shapes which is in agreement with DLS analysis (Fig. 10.b). Based on the TEM observations as well as assuming full conversion of the precursor, we estimate the number of aggregates that form in each droplet to around 400. These aggregates consist of randomly ordered primary nanoparticles with a rod-like morphology and a size of $10 \mathrm{~nm}$ in the $k$ direction as well as a size of about $20 \mathrm{~nm}$ in the $l$ direction (inset in Fig. 10.b). This is in good accordance with the results obtained from powder X-ray diffraction (XRD), where we determined a crystallite size of $10.9 \mathrm{~nm}$ for the $k$ direction from the (010) reflection and a crystallite size of $21.9 \mathrm{~nm}$ for the $l$ direction from the (002) reflection of $\mathrm{ZnO}$ (Fig. 11) with a hexagonal lattice structure (reference pattern ICSD 98-0057450). Rod-like $\mathrm{ZnO}$ nanoparticles are known to form easily due to the hexagonal crystal structure, 
and have previously been reported to result from syntheses under the influence of ligand species (Zhang et al., 2006) such as in the nonaqueous reaction of zinc acetylacetonate hydrate in benzyl alcohol (Zellmer et al., 2015). As we obtain this characteristic morphology, one can assume that the droplet containing the reaction mixture was successfully used as a micro-reactor. However, one must not underestimate the role of the fluorocarbon based surfactant and DP which seem to have a dominant impact on the assembly of the aggregates. Possibly, the surfactant stabilizes the aggregates against further aggregation, resulting in the observed structures of about $150 \mathrm{~nm}$ average size.
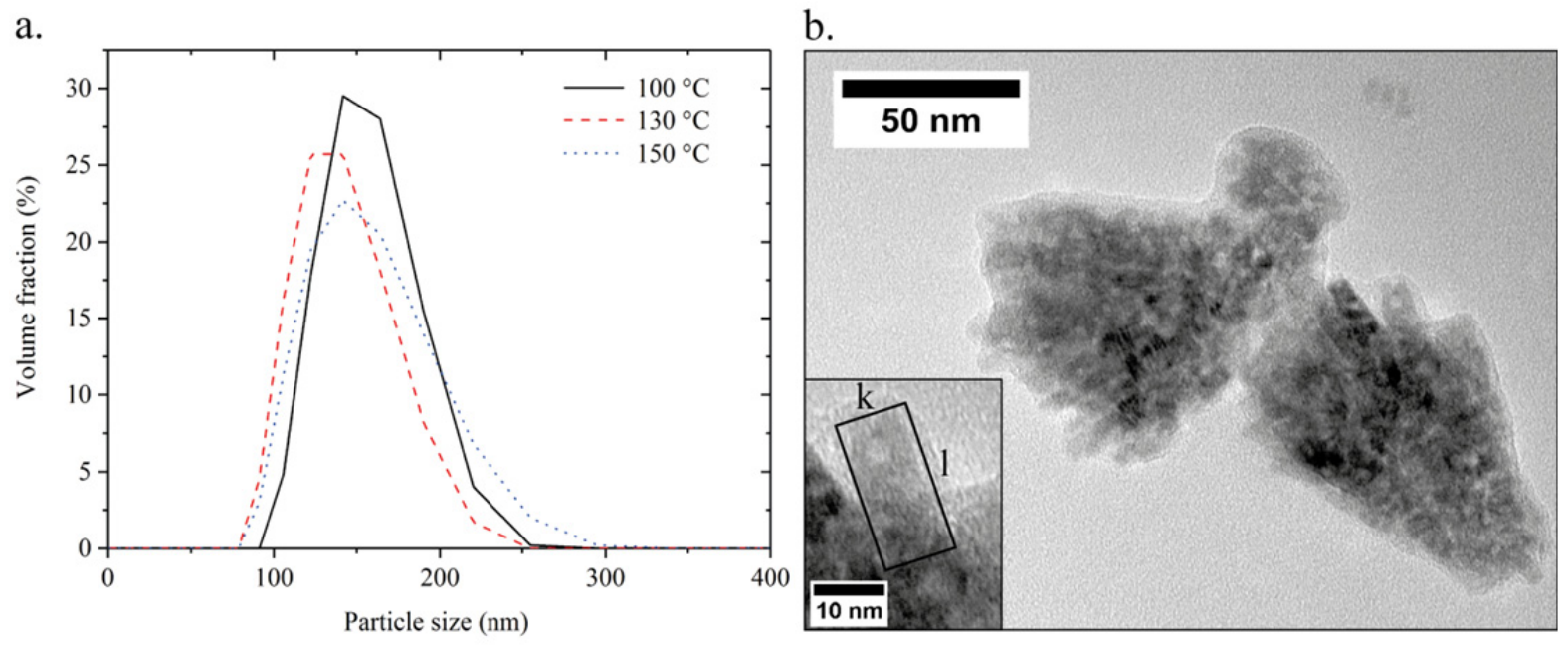

Fig. 10: a. Particle size distributions of $\mathrm{ZnO}$ aggregates synthesized at various temperatures as obtained by DLS; b. Transmission electron micrograph of randomly shaped $\mathrm{ZnO}$ aggregates assembled from rod-like primary particles (inset) synthesized at $130^{\circ} \mathrm{C}$.

This is supported by the observation that the solid and liquid phases could not be separated by centrifugation. Thus, we had to dry the sample under vacuum at $40^{\circ} \mathrm{C}$ for 24 hours to recover the nanoparticles in order to perform XRD analyses (Fig. 11), which resulted in a high amount of organics within the sample, explaining the broad reflections in the diffractogram. Furthermore, additional reflections are visible at $39^{\circ}$ and $52.5^{\circ} 2 \Theta$ in the diffraction pattern (Fig. 11), which we attribute to 
from small amounts of beta-zinc hydroxide (ICSD 98-002-0285) as side product of this synthesis.

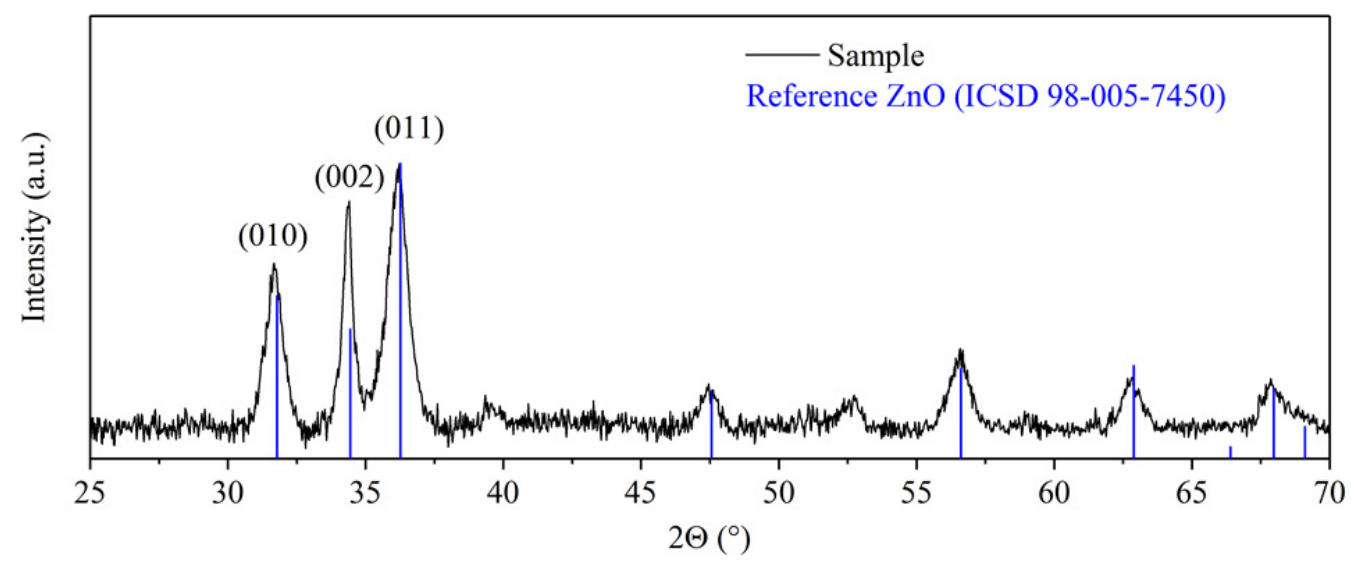

Fig. 11: Powder $\mathrm{XRD}$ pattern of a $\mathrm{ZnO}$ sample synthesized at $130{ }^{\circ} \mathrm{C}$ indicating a rod-like structure of the crystallites and the corresponding reference pattern of the hexagonal ZnO polymorph (ICSD code:98-005-7450).

\subsection{Synthesis of $\mathrm{CeO}_{2}$ nanoparticles}

The $\mathrm{CeO}_{2}$ nanoparticle synthesis was carried out in the meandering channel system as well as in the expansion channel - SBR system at a temperature of $170{ }^{\circ} \mathrm{C}$, using a precursor concentration of $180 \mathrm{mmol} \mathrm{L}^{-1}$. Similar to the $\mathrm{ZnO}$ model system, the solid precursor species cerium trichloride was dissolved in benzyl alcohol before the synthesis to prevent clogging of the tubes and the emulsification zone during longer experiments.

Both microfluidic reaction systems yielded round primary particles with a size of $6 \mathrm{~nm}$ (Fig. 12.a and Fig. 12.b) which assembled, like the $\mathrm{TiO}_{2}$ system, to spherical aggregate structures. In contrast, the $\mathrm{CeO}_{2}$ aggregates possessed a smaller size of $100 \mathrm{~nm}$, and additionally many individual primary particles are present in the product suspension. We performed a selected area electron diffraction on the primary nanoparticles and found diffraction spots corresponding to the characteristic $\mathrm{CeO}_{2}$ pattern which enabled us to identify the reflections of the (111) and (002) lattice planes as illustrated in Fig. 12.c. 


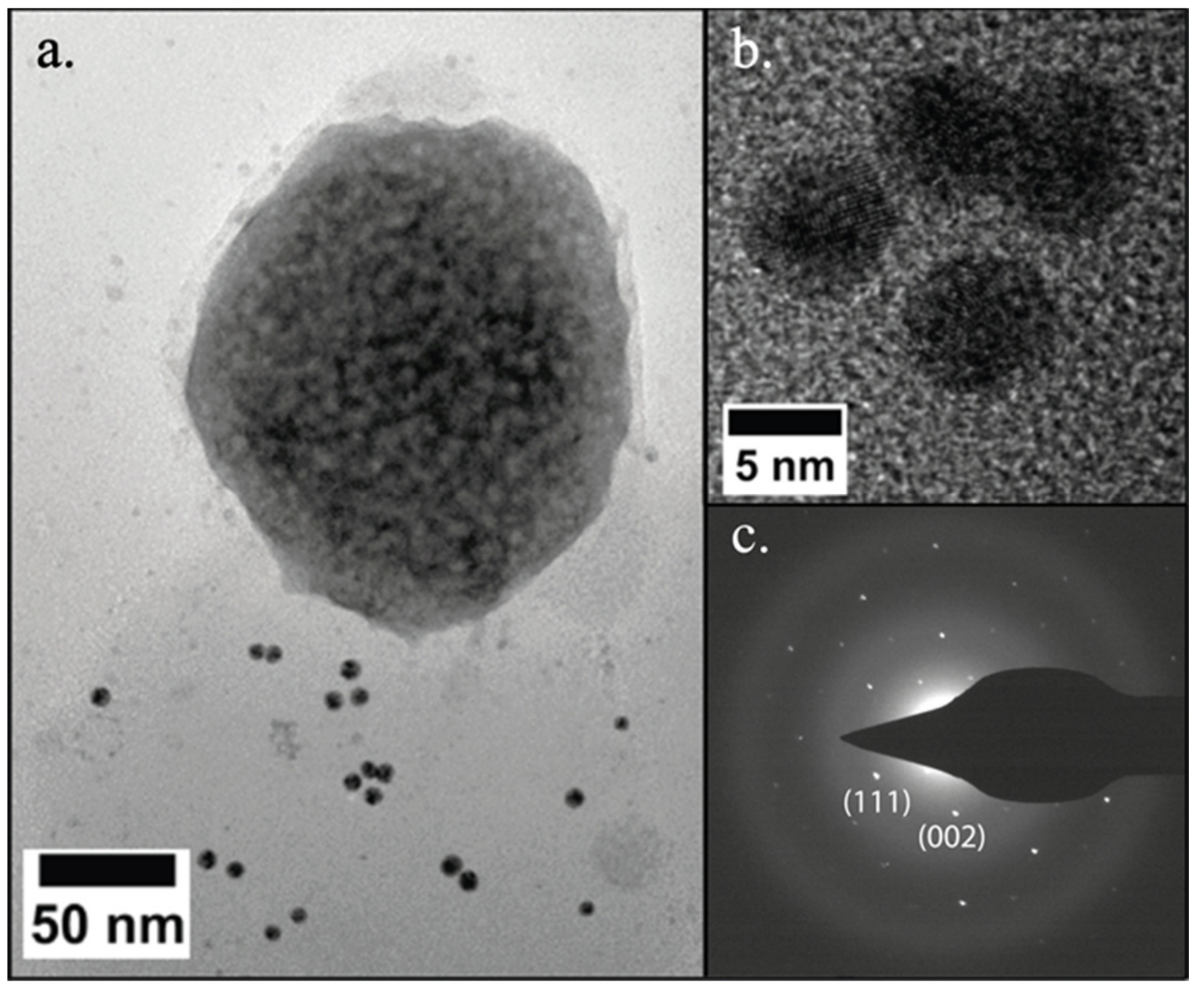

Fig. 12: Transmission electron microscopy of a $\mathrm{CeO}_{2}$ sample synthesized at $170{ }^{\circ} \mathrm{C}$; a. shows a spherical aggregate structure and stable primary nanoparticles; b. shows primary $\mathrm{CeO}_{2}$ nanoparticles; c. selected area electron diffraction of a $\mathrm{CeO}_{2}$ nanoparticle illustrating the (111) and (002) lattice planes.

The high crystallinity of the nanoparticles was confirmed by XRD analysis (Fig. 13) which shows that the reflection of the (111) plane is most dominant. We used this reflection to determine a crystallite size of $7.8 \mathrm{~nm}$ which is slightly larger than the size of $6 \mathrm{~nm}$ obtained by TEM. Similar grain sizes were reported by Rebellato et al. who used a microemulsion technique (Rebellato et al., 2008) to synthesize ceria nanoparticles. However, no evidence of the formation of spherical aggregates was found, which indicates that the characteristic globular structure of the aggregates obtained in our study is again provoked by the dissolution of the reaction mixture into the continuous phase fluid. Yet, the diffractogram exhibits deviations from the reference pattern (ICSD 98-015-5610) in a similar fashion 
as the $\mathrm{ZnO}$ system, such as slightly shifted reflections and additional signals at $23^{\circ}$ and $44^{\circ} 2 \Theta$. These signals possibly result from small amounts of unreacted cerium chloride (ICSD 98-003-1575). Nevertheless, the fluorocarbon surfactant and the continuous phase fluid seem to have a great impact on the particle formation reaction and hence on the $\mathrm{CeO}_{2}$ lattice structure. The influence of organics during a ceria nanoparticle synthesis was previously shown by Moreno et al. who used a chemical bath deposition method with a subsequent thermal annealing step. They found contaminations of organic groups (Moreno et al., 2017) within the nanoparticle product through FTIR-analysis and showed these contaminations disappear after annealing. Nevertheless, the fabrication of ceria nanoparticles from solid state precursor is possible with the investigated microfluidic synthesis concepts and can be conducted under stable conditions for longer operation times.

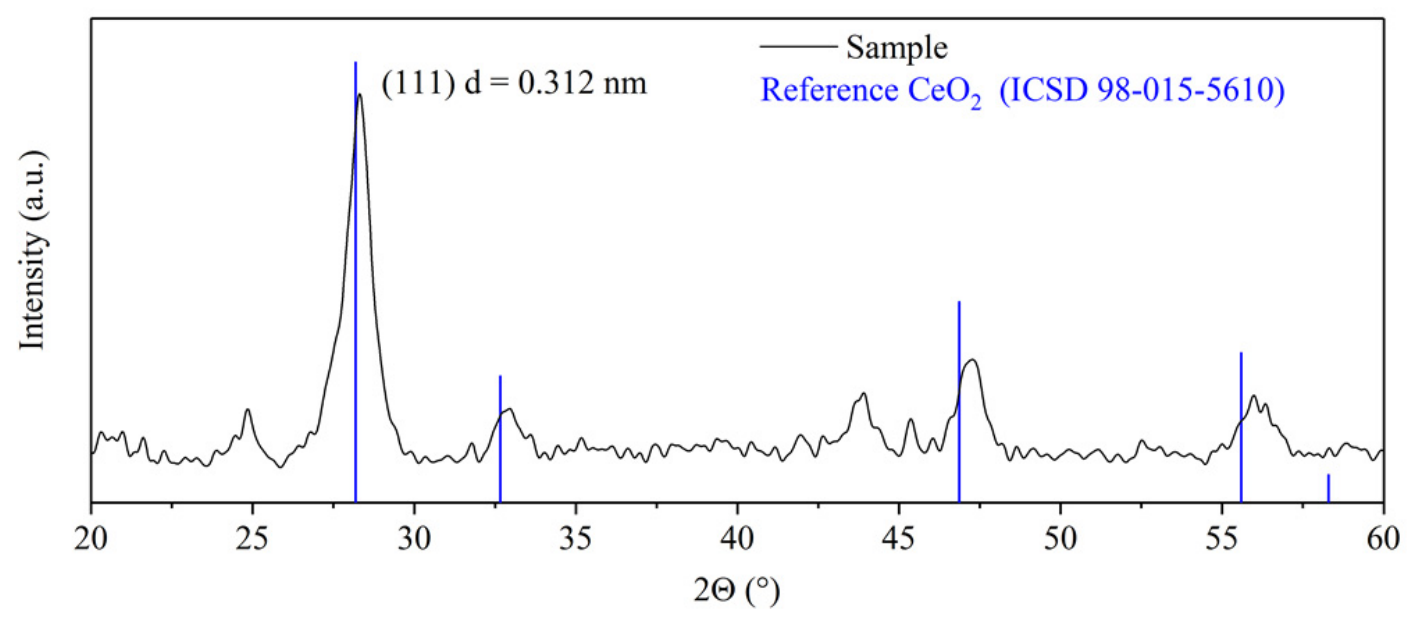

Fig. 13: $\mathrm{XRD}$ analysis of a sample obtained from a $\mathrm{CeO}_{2}$ synthesis at $170{ }^{\circ} \mathrm{C}$, showing slightly shifted as well as some additional reflections when compared to the $\mathrm{CeO}_{2}$ reference pattern (ICSD 98 015-5610).

\section{Conclusion}

We have presented a droplet based microfluidic synthesis concept to fabricate various nanostructured metal oxides via the nonaqueous sol-gel method. Two different microfluidic systems with locally heatable reaction zone areas were investigated. The systems allowed precise and fast control of the applied operation pressures and therefore enabled stable droplet formation with different fluids as well as changing viscosities due to heating. Furthermore, easy tuning of the droplet size was 
possible by adjusting the pressure ratio between the continuous and disperse phases. Temperature was precisely set using a control loop integrated to the mounting. The systems allow for the usage of solid as well as liquid precursor species and are suitable for syntheses with short and long reaction times. We found that the reaction medium is miscible with the continuous phase fluid at elevated temperatures, which leads to the formation of spherical aggregate structures when syntheses are conducted above $150{ }^{\circ} \mathrm{C}$. We investigated the synthesis of $\mathrm{TiO}_{2}$ nanoparticles and showed that the synthesis can be carried out in microfluidic systems designed for longer reaction times. The systems yielded highly spherical aggregate structures of $13.5 \pm 3.5 \mu \mathrm{m}$ due to the dissolution of the reaction medium with highly crystalline primary nanoparticles with a size of $6.5 \mathrm{~nm}$. In contrast, the $\mathrm{ZnO}$ syntheses were solely performed in a microfluidic expansion channel system with short droplet residence times at lower temperatures. A solid precursor species was used which necessitated a previous dissolution step at $70-80^{\circ} \mathrm{C}$ in the solvent benzyl alcohol. As no droplet shrinkage occurred at these lower temperatures, the microfluidic droplet served as the reaction volume in which the selfassembly of randomly shaped aggregates with a size of $150 \mathrm{~nm}$ occurred. The $\mathrm{CeO}_{2}$ syntheses comprised the usage of a solid precursor species in combination with higher temperatures and still required longer reaction times to initiate particle formation. With respect to these demands, the microfluidic systems designed for long droplet residence times were operated under stable and steady conditions over the course of longer experiments. This resulted in spherical primary nanoparticles which assembled to globular aggregate structures in a similar fashion to the $\mathrm{TiO}_{2}$ system, however being much smaller in size. For theTiO, $\mathrm{ZnO}$ as well as the $\mathrm{CeO}_{2}$ synthesis, we observed the influence of the high organic content as well as the fluorinated surfactant species, leading to the formation of characteristic aggregate structures and thereby having a major impact on the final product properties. One has to take into account that the mass quantity of the resulting nanoparticles was very low. Thus, the preparation of higher quantities necessitates subsequent post-processing steps such as solvent removal and particle washing, possibly increasing the final product quality. This could be achieved by a scale-up through the parallelization of a number of microfluidic systems, which is feasible since we already have shown steady operation of single systems over longer periods. 


\section{Acknowledgments}

We acknowledge financial support from "Niedersächsisches Vorab" through the "Quantum- and Nano-Metrology (QUANOMET)" initiative within the project NP-1. The authors would furthermore like to thank Bilal Temel for TEM, SAED and XRD measurements. We also acknowledge the Laboratory of Nano and Quantum Engineering (LNQE), Leibniz University Hannover, for use of the TEM instrument. Moreover, the authors are grateful to Ramazan Aydemir for great experimental assistance

\section{References}

Anna, S.L., Bontoux, N., Stone, H.A., 2003. Formation of dispersions using "flow focusing" in microchannels. Applied Physics Letters 82, 364-366.

Barth, N., Zimmermann, M., Becker, A.E., Graumann, T., Garnweitner, G., Kwade, A., 2015. Influence of $\mathrm{TiO}_{2}$ nanoparticle synthesis on the properties of thin coatings. Thin Solid Films 574, 20-27.

Bayani, M., Ehsani, M., Khonakdar, H.A., Seyfi, J., HosseinAbadi-Ghaeni, M.H., 2016. An investigation of $\mathrm{TiO}_{2}$ nanoparticles effect on morphology, thermal, and mechanical properties of epoxy/silica composites. Journal of Vinyl and Additive Technology 23, E216-E221.

Bilecka, I., Luo, L., Djerdj, I., Rossell, M.D., Jagodič, M., Jagličić, Z., Masubuchi, Y., Kikkawa, S., Niederberger, M., 2011. Microwave-Assisted Nonaqueous Sol-Gel Chemistry for Highly Concentrated ZnO-Based Magnetic Semiconductor Nanocrystals. The Journal of Physical Chemistry C 115, 1484-1495.

Bills, B., Morris, N., Dubey, M., Wang, Q., Fan, Q.H., 2015. Electrophoretic deposited $\mathrm{TiO}_{2}$ pigmentbased back reflectors for thin film solar cells. Optics Express 23, A71-A82.

Carroll, N.J., Rathod, S.B., Derbins, E., Mendez, S., Weitz, D.A., Petsev, D.N., 2008. Droplet-Based Microfluidics for Emulsion and Solvent Evaporation Synthesis of Monodisperse Mesoporous Silica Microspheres. Langmuir 24, 658-661.

Chang, C.-H., Paul, B.K., Remcho, V.T., Atre, S., Hutchison, J.E., 2008. Synthesis and postprocessing of nanomaterials using microreaction technology. Journal of Nanoparticle Research 10, 965-980.

Cheema, T., Lichtner, A., Weichert, C., Böl, M., Garnweitner, G., 2012. Fabrication of transparent polymer-matrix nanocomposites with enhanced mechanical properties from chemically modified $\mathrm{ZrO} 2$ nanoparticles. Journal of Materials Science 47, 2665-2674.

Chen, G., Xu, Y., 2018. Biosynthesis of cerium oxide nanoparticles and their effect on lipopolysaccharide (LPS) induced sepsis mortality and associated hepatic dysfunction in male Sprague Dawley rats. Materials Science and Engineering: C 83, 148-153.

Cottam, B.F., Krishnadasan, S., deMello, A.J., deMello, J.C., Shaffer, M.S.P., 2007. Accelerated synthesis of titanium oxide nanostructures using microfluidic chips. Lab on a Chip 7, 167-169.

Deshpande, A.S., Pinna, N., Smarsly, B., Antonietti, M., Niederberger, M., 2005. Controlled Assembly of Preformed Ceria Nanocrystals into Highly Ordered 3D Nanostructures. Small 1, 313316. 
Erfle, P., Riewe, J., Bunjes, H., Dietzel, A., 2017. Optically monitored segmented flow for controlled ultra-fast mixing and nanoparticle precipitation. Microfluidics and Nanofluidics 21, 179.

Fischer, M.G., Hua, X., Wilts, B.D., Gunkel, I., Bennett, T.M., Steiner, U., 2017. Mesoporous Titania Microspheres with Highly Tunable Pores as an Anode Material for Lithium Ion Batteries. ACS Applied Materials \& Interfaces 9, 22388-22397.

Garnweitner, G., Niederberger, M., 2008. Organic chemistry in inorganic nanomaterials synthesis. Journal of Materials Chemistry 18, 1171-1182.

Garnweitner, G., Tsedev, N., Dierke, H., Niederberger, M., 2008. Benzylamines as Versatile Agents for the One-Pot Synthesis and Highly Ordered Stacking of Anatase Nanoplatelets. European Journal of Inorganic Chemistry 2008, 890-895.

Gröhn, A.J., Pratsinis, S.E., Sánchez-Ferrer, A., Mezzenga, R., Wegner, K., 2014. Scale-up of Nanoparticle Synthesis by Flame Spray Pyrolysis: The High-Temperature Particle Residence Time. Industrial \& Engineering Chemistry Research 53, 10734-10742.

Gruar, R.I., Tighe, C.J., Darr, J.A., 2013. Scaling-up a Confined Jet Reactor for the Continuous Hydrothermal Manufacture of Nanomaterials. Industrial \& Engineering Chemistry Research 52, $5270-5281$.

Ha, N.H., Thinh, D.D., Huong, N.T., Phuong, N.H., Thach, P.D., Hong, H.S., 2018. Fast response of carbon monoxide gas sensors using a highly porous network of $\mathrm{ZnO}$ nanoparticles decorated on $3 \mathrm{D}$ reduced graphene oxide. Applied Surface Science 434, 1048-1054.

Holtze, C., Rowat, A.C., Agresti, J.J., Hutchison, J.B., Angile, F.E., Schmitz, C.H.J., Koster, S., Duan, H., Humphry, K.J., Scanga, R.A., Johnson, J.S., Pisignano, D., Weitz, D.A., 2008. Biocompatible surfactants for water-in-fluorocarbon emulsions. Lab on a Chip 8, 1632-1639.

Jensen, G.V., Bremholm, M., Lock, N., Deen, G.R., Jensen, T.R., Iversen, B.B., Niederberger, M., Pedersen, J.S., Birkedal, H., 2010. Anisotropic Crystal Growth Kinetics of Anatase $\mathrm{TiO}_{2}$ Nanoparticles Synthesized in a Nonaqueous Medium. Chemistry of Materials 22, 6044-6055.

Jossen, R., Heine, M.C., Pratsinis, S.E., Akhtar, M.K., 2006. Thermal stability of flame-made zirconia-based mixed oxides. Chemical Vapor Deposition 12, 614-619.

Khan, S.A., Günther, A., Schmidt, M.A., Jensen, K.F., 2004. Microfluidic Synthesis of Colloidal Silica. Langmuir 20, 8604-8611.

Khataee, A., Gholami, P., Kalderis, D., Pachatouridou, E., Konsolakis, M., 2018. Preparation of novel $\mathrm{CeO}_{2}$-biochar nanocomposite for sonocatalytic degradation of a textile dye. Ultrasonics Sonochemistry 41, 503-513.

Koczkur, K.M., Mourdikoudis, S., Polavarapu, L., Skrabalak, S.E., 2015. Polyvinylpyrrolidone (PVP) in nanoparticle synthesis. Dalton Transactions 44, 17883-17905.

Koziej, D., Floryan, C., Sperling, R.A., Ehrlicher, A.J., Issadore, D., Westervelt, R., Weitz, D.A., 2013. Microwave dielectric heating of non-aqueous droplets in a microfluidic device for nanoparticle synthesis. Nanoscale 5, 5468-5475.

Lee, M., Collins, J.W., Aubrecht, D.M., Sperling, R.A., Solomon, L., Ha, J.-W., Yi, G.-R., Weitz, D.A., Manoharan, V.N., 2014. Synchronized reinjection and coalescence of droplets in microfluidics. Lab on a Chip 14, 509-513.

Lee, S.-K., Liu, X., Sebastian Cabeza, V., Jensen, K.F., 2012. Synthesis, assembly and reaction of a nanocatalyst in microfluidic systems: a general platform. Lab on a Chip 12, 4080-4084.

Lorenz, T., Bojko, S., Bunjes, H., Dietzel, A., 2018. An inert 3D emulsification device for individual precipitation and concentration of amorphous drug nanoparticles. Lab on a Chip.

Low, J., Qiu, S., Xu, D., Jiang, C., Cheng, B., 2018. Direct evidence and enhancement of surface plasmon resonance effect on Ag-loaded $\mathrm{TiO}_{2}$ nanotube arrays for photocatalytic $\mathrm{CO}_{2}$ reduction. Applied Surface Science 434, 423-432.

Lu, D., Gao, Q., Wu, X., Fan, Y., 2017. ZnO nanostructures decorated hollow glass microspheres as near infrared reflective pigment. Ceramics International 43, 9164-9170.

Ludi, B., Suess, M.J., Werner, I.A., Niederberger, M., 2012. Mechanistic aspects of molecular formation and crystallization of zinc oxide nanoparticles in benzyl alcohol. Nanoscale 4, 19821995.

Luo, L., Häfliger, K., Xie, D., Niederberger, M., 2013. Transparent conducting Sn: ZnO films deposited from nanoparticles. Journal of Sol-Gel Science and Technology 65, 28-35. 
Mallakpour, S., Shamsaddinimotlagh, S., 2018. Ultrasonic-promoted rapid preparation of $\mathrm{PVC} / \mathrm{TiO}_{2^{-}}$ BSA nanocomposites: Characterization and photocatalytic degradation of methylene blue. Ultrasonics Sonochemistry 41, 361-374.

Marre, S., Park, J., Rempel, J., Guan, J., Bawendi Moungi, G., Jensen Klavs, F., 2008. Supercritical Continuous-Microflow Synthesis of Narrow Size Distribution Quantum Dots. Advanced Materials 20, 4830-4834.

McDonald, J.C., Whitesides, G.M., 2002. Poly(dimethylsiloxane) as a Material for Fabricating Microfluidic Devices. Accounts of Chemical Research 35, 491-499.

Moreno, O.P., Pérez, R.G., Merino, R.P., Portillo, M.C., Tellez, G.H., Rosas, E.R., Tototzintle, M.Z., 2017. $\mathrm{CeO}_{2}$ nanoparticles growth by chemical bath and its thermal annealing treatment in air atmosphere. Optik - International Journal for Light and Electron Optics 148, 142-150.

Mueller, R., Mädler, L., Pratsinis, S.E., 2003. Nanoparticle synthesis at high production rates by flame spray pyrolysis. Chemical Engineering Science 58, 1969-1976.

Niederberger, M., Bartl, M.H., Stucky, G.D., 2002. Benzyl Alcohol and Transition Metal Chlorides as a Versatile Reaction System for the Nonaqueous and Low-Temperature Synthesis of Crystalline Nano-Objects with Controlled Dimensionality. Journal of the American Chemical Society 124, 13642-13643.

Niederberger, M., Garnweitner, G., Pinna, N., Neri, G., 2005. Non-aqueous routes to crystalline metal oxide nanoparticles: Formation mechanisms and applications. Progress in Solid State Chemistry $33,59-70$.

Nightingale, A.M., Krishnadasan, S.H., Berhanu, D., Niu, X., Drury, C., McIntyre, R., Valsami-Jones, E., deMello, J.C., 2011. A stable droplet reactor for high temperature nanocrystal synthesis. Lab on a Chip 11, 1221-1227.

Nishioka, M., Miyakawa, M., Kataoka, H., Koda, H., Sato, K., Suzuki, T.M., 2011. Continuous synthesis of monodispersed silver nanoparticles using a homogeneous heating microwave reactor system. Nanoscale 3, 2621-2626.

Olliges-Stadler, I., Rossell, M.D., Suess, M.J., Ludi, B., Bunk, O., Pedersen, J.S., Birkedal, H., Niederberger, M., 2013. A comprehensive study of the crystallization mechanism involved in the nonaqueous formation of tungstite. Nanoscale 5, 8517-8525.

Pinna, N., Karmaoui, M., Willinger, M.-G., 2011. The "benzyl alcohol route": An elegant approach towards doped and multimetal oxide nanocrystals. Journal of Sol-Gel Science and Technology 57, 323-329.

Ralf, S., Martin, B., Thomas, P., Stephan, H., 2012. Droplet based microfluidics. Reports on Progress in Physics 75, 016601.

Rebellato, J., Natile, M.M., Glisenti, A., 2008. Influence of the synthesis procedure on the properties and reactivity of nanostructured ceria powders. Applied Catalysis A: General 339, 108-120.

Ruusunen, J., Pyykönen, J., Ihalainen, M., Tiitta, P., Torvela, T., Karhunen, T., Sippula, O., Qin, Q.H., van Dijken, S., Joutsensaari, J., Lähde, A., Jokiniemi, J., 2015. A Novel Porous Tube Reactor for Nanoparticle Synthesis with Simultaneous Gas-Phase Reaction and Dilution. Aerosol Science and Technology 49, 1170-1180.

Scott, R.L., 1948. The Solubility of Fluorocarbons1. Journal of the American Chemical Society 70, 4090-4093.

Segets, D., Gradl, J., Taylor, R.K., Vassilev, V., Peukert, W., 2009. Analysis of Optical Absorbance Spectra for the Determination of ZnO Nanoparticle Size Distribution, Solubility, and Surface Energy. ACS Nano 3, 1703-1710.

Seong, G., Dejhosseini, M., Adschiri, T., 2018. A kinetic study of catalytic hydrothermal reactions of acetaldehyde with cubic $\mathrm{CeO}_{2}$ nanoparticles. Applied Catalysis A: General 550, 284-296.

Shi, H.-h., Xiao, Y., Ferguson, S., Huang, X., Wang, N., Hao, H.-x., 2017. Progress of crystallization in microfluidic devices. Lab on a Chip 17, 2167-2185.

Slostowski, C., Marre, S., Babot, O., Toupance, T., Aymonier, C., 2012. Near- and Supercritical Alcohols as Solvents and Surface Modifiers for the Continuous Synthesis of Cerium Oxide Nanoparticles. Langmuir 28, 16656-16663.

Song, H., Chen, D.L., Ismagilov, R.F., 2006. Reactions in Droplets in Microfluidic Channels. Angewandte Chemie International Edition 45, 7336-7356. 
Song, H., Wu, R., Yang, J., Dong, J., Ji, G., 2018. Fabrication of $\mathrm{CeO}_{2}$ nanoparticles decorated threedimensional flower-like BiOI composites to build p-n heterojunction with highly enhanced visiblelight photocatalytic performance. Journal of Colloid and Interface Science 512, 325-334.

Sophia Lee, S.H., Dawood, M.K., Choi, W.K., Alan Hatton, T., Khan, S.A., 2012. Hierarchical materials synthesis at soft all-aqueous interfaces. Soft Matter 8, 3924-3928.

Stolzenburg, P., Garnweitner, G., 2017. Experimental and numerical insights into the formation of zirconia nanoparticles: a population balance model for the nonaqueous synthesis. Reaction Chemistry \& Engineering 2, 337-348.

Tsuzuki, T., 2009. Commercial scale production of inorganic nanoparticles. International Journal of Nanotechnology 6, 567-578.

Uzunoglu, A., Stanciu, L.A., 2016. Novel $\mathrm{CeO}_{2}-\mathrm{CuO}$-decorated enzymatic lactate biosensors operating in low oxygen environments. Analytica Chimica Acta 909, 121-128.

Wang, J., Li, Y., Wang, X., Wang, J., Tian, H., Zhao, P., Tian, Y., Gu, Y., Wang, L., Wang, C., 2017 a. Droplet Microfluidics for the Production of Microparticles and Nanoparticles. Micromachines 8, 22.

Wang, X., Jia, J., Wang, Y., 2017b. Combination of photocatalysis with hydrodynamic cavitation for degradation of tetracycline. Chemical Engineering Journal 315, 274-282.

Yen, B.K.H., Günther, A., Schmidt, M.A., Jensen, K.F., Bawendi, M.G., 2005. A Microfabricated Gas-Liquid Segmented Flow Reactor for High-Temperature Synthesis: The Case of CdSe Quantum Dots. Angewandte Chemie International Edition 44, 5447-5451.

Zellmer, S., Kockmann, A., Dosch, I., Temel, B., Garnweitner, G., 2015. Aluminum zinc oxide nanostructures with customized size and shape by non-aqueous synthesis. CrystEngComm 17, 6878-6883.

Zhang, M., Wang, W., Xie, R., Ju, X., Liu, Z., Jiang, L., Chen, Q., Chu, L., 2016. Controllable microfluidic strategies for fabricating microparticles using emulsions as templates. Particuology 24, $18-31$.

Zhang, T., Dong, W., Keeter-Brewer, M., Konar, S., Njabon, R.N., Tian, Z.R., 2006. Site-Specific Nucleation and Growth Kinetics in Hierarchical Nanosyntheses of Branched ZnO Crystallites. Journal of the American Chemical Society 128, 10960-10968.

Zhao, C.-X., Middelberg, A.P.J., 2014. Titania microparticles using a facile microfluidic mass-transfer control method. Chemical Engineering Science 112, 10-14.

Zimmermann, M., Garnweitner, G., 2012. Spontaneous water release inducing nucleation during the nonaqueous synthesis of $\mathrm{TiO}_{2}$ nanoparticles. CrystEngComm 14, 8562-8568.

Zimmermann, M., Temel, B., Garnweitner, G., 2013. Parameter studies of the synthesis of titanium dioxide nanoparticles: Effect on particle formation and size. Chemical Engineering and Processing: Process Intensification 74, 83-89. 